\title{
Stability and Stabilization of Boolean Networks *
}

\author{
Daizhan Cheng ${ }^{\dagger}$, Hongsheng $\mathrm{Qi}^{\dagger}$, Zhiqiang $\mathrm{Li}^{\dagger}$, Jiang B. Liu ${ }^{\ddagger *}$ \\ $\dagger$ Laboratory of Systems and Control, AMSS, Chinese Academy of Sciences, Beijing 100190, China \\ E-mail: dcheng@iss.ac.cn, qihongsh@amss.ac.cn, lizhiqiang@amss.ac.cn \\ $¥$ State Key Laboratory of Software Engineering, Wuhan Univ., Wuhan 430072, China \\ * Dept. of Comp. Sci. \& Inform. Sys., Bradley University, Peoria, IL, USA \\ E-mail: jiangbo@bumail.bradley.edu \\ (Corresponding Author)
}

\begin{abstract}
The stability of Boolean networks and the stabilization of Boolean control networks are investigated. Using semi-tensor product of matrices and the matrix expression of logic, the dynamics of a Boolean (control) network can be converted to a discrete time linear (bilinear) dynamics, called the algebraic form of the Boolean (control) network. Then the stability can be revealed by analyzing the transition matrix of the corresponding discrete time system. Main results consist of two parts: (i) Using logic coordinate transformation, the known sufficient condition based on incidence matrix has been improved. It can also be used in stabilizer design. (ii) Based on algebraic form, necessary and sufficient conditions for stability and stabilization respectively are obtained.
\end{abstract}

Key Words. Boolean (control) system, stability, stabilization, semi-tensor product of matrices.

\section{Introduction}

Accompanying the rising of systems biology, the study of Boolean network has attracted a great attention from biologists, physicists, and systems scientists, because it is a proper model to describe cellular networks and genomic regulatory networks $[10,7]$. The control of Boolean network is a challenging problem. So far, there are only few results because we are short of systematic tool to deal with logical dynamic systems $[1,8]$.

Recently, a new matrix product, namely, the semi-tensor product (STP) of matrices, was proposed, which generalizes the conventional matrix product, $A B, A \in M_{p \times q}$ and $B \in M_{m \times n}$ for the case $q \neq m$, where $M_{m \times n}$ stands for the set of $m \times n$ matrices. Using STP, a logical equation can be expressed as an algebraic equation and the dynamics of a Boolean (control) network can be converted into a linear (bilinear) discrete-time (control) system [2]. Then investigating the corresponding discrete time (control) systems, some interesting progresses have been achieved, which are briefly described as follows: (1) Formulas for calculating fixed points and cycles have been obtained [3]; (ii) Steering gears' structure of attractors in Boolean network has been revealed [4], which explains why tiny attractors decide the vast order, as described in [11]; (iii) The controllability and observability of Boolean control networks

*Supported partly by NNSF 60674022, 60736022, and 60821091 of China. 
are investigated [5]; (iv) Coordinate transformation of Boolean networks and the realization of Boolean control networks are presented [6].

Stability of Boolean networks is another interesting topic [9, 12]. In fact, the stability of a Boolean network is essentially equivalent to the convergence of a discrete iteration [14]. As for the stabilization of a Boolean control network, to the best of authors' knowledge, it is a new topic.

This paper considers the stability of Boolean networks and the stabilization of Boolean control networks. The key tool used in this paper is the semi-tensor product of matrices and the matrix expression of logic, which have been used in the sequence of works $[3,4,5,6]$.

The paper is organized as follows. Section 2 provides necessary preliminaries, including (i) matrix expression of logic; (ii) Boolean (control) network; (iii) Logical coordinate transformation; (iv) incidence matrix. Section 3 discusses the concepts and basic properties of Boolean matrices. The vector distance of Boolean matrices is also introduced. The global stability of Boolean networks is investigated in Section 4. Based on the incidence matrix and the vector distance of Boolean matrices, a convenient sufficient condition is obtained. Moreover, the necessary and sufficient condition is also presented. Section 5 considers the stabilization of Boolean control networks. Some examples are included to depict the technique. Section 6 is the conclusion.

\section{Preliminaries}

\subsection{Matrix Expression of Logic}

We first recall the definition of STP of matrices.

Definition 2.1 [2] 1. Let $X$ be a row vector of dimension np, and $Y$ be a column vector with dimension p. Then we split $X$ into $p$ equal-size blocks as $X^{1}, \cdots, X^{p}$, which are $1 \times n$ rows. Define the $S T P$, denoted by $\ltimes$, as

$$
\left\{\begin{array}{l}
X \ltimes Y=\sum_{i=1}^{p} X^{i} y_{i} \in \mathbb{R}^{n}, \\
Y^{T} \ltimes X^{T}=\sum_{i=1}^{p} y_{i}\left(X^{i}\right)^{T} \in \mathbb{R}^{n} .
\end{array}\right.
$$

2. Let $A \in M_{m \times n}$ and $B \in M_{p \times q}$. If either $n$ is a factor of $p$, say $n t=p$ and denote it as $A \prec_{t} B$, or $p$ is a factor of $n$, say $n=p t$ and denote it as $A \succ_{t} B$, then we define the STP of $A$ and $B$, denoted by $C=A \ltimes B$, as the following: $C$ consists of $m \times q$ blocks as $C=\left(C^{i j}\right)$ and each block is

$$
C^{i j}=A^{i} \ltimes B_{j}, \quad i=1, \cdots, m, \quad j=1, \cdots, q,
$$

where $A^{i}$ is the $i$-th row of $A$ and $B_{j}$ is the $j$-th column of $B$.

STP of matrices is a generalization of conventional matrix product. So we can omit the symbol $\ltimes$. All fundamental properties of conventional matrix product remain true [2]. Throughout this paper the matrix products are assumed to be semi-tensor product. As $n=p$ they becomes conventional matrix product automatically.

We briefly define some concepts and/or notations first. 
- Let $\delta_{n}^{i}$ be the $i$-th column of the identity matrix $I_{n}$, and $\Delta_{n}:=\left\{\delta_{n}^{1}, \delta_{n}^{2}, \cdots, \delta_{n}^{n}\right\}$. When $n=2$ we simply use $\Delta:=\Delta_{2}$.

- The set of logical values: True $(T \sim 1)$ and False $(F \sim 0)$, is denoted by $\mathcal{D}=\{0,1\}$. We identify each with a vector as $T \sim \delta_{2}^{1}$ and $F \sim \delta_{2}^{2}$. So in vector form the set of logical values becomes $\Delta$. In this sense we have the equivalence as $\mathcal{D} \sim \Delta$.

- Assume a matrix $M=\left[\begin{array}{llll}\delta_{n}^{i_{1}} & \delta_{n}^{i_{2}} & \cdots & \delta_{n}^{i_{s}}\end{array}\right] \in M_{n \times s}$, i.e., its columns, $\operatorname{Col}(M) \subset \Delta_{n}$. We call $M$ a logical matrix, and simply denote it as

$$
M=\delta_{n}\left[\begin{array}{llll}
i_{1} & i_{2} & \cdots & i_{s}
\end{array}\right]
$$

- The set of $n \times s$ logical matrices is denoted by $\mathcal{L}_{n \times s}$.

- A matrix $B \in M_{n \times s}$ is called a Boolean matrix, if its entries $b_{i j} \in \mathcal{D}, \forall i, j$.

- The set of $n \times s$ Boolean matrices is denoted by $\mathcal{B}_{n \times s}$.

In vector form, we have

Proposition 2.2 [2] Let $x_{1}, \cdots, x_{n} \in \mathcal{D}$ be $n$ logical variables, and $f\left(x_{1}, \cdots, x_{n}\right)$ a logical function. Then there exists a unique matrix $M_{f} \in \mathcal{L}_{2 \times 2^{n}}$, called the structure matrix of $f$, such that in vector form we have

$$
f\left(x_{1}, \cdots, x_{n}\right)=M_{f} \ltimes_{i=1}^{n} x_{i}, \quad x_{i} \in \Delta .
$$

Logical operators are fundamental logical functions. Their structure matrices are particularly useful. In the following table we list the structure matrices for some basic logical operators (Negation: $\neg$; Conjunction: $\wedge$; Disjunction: $\vee$; Conditional: $\rightarrow$; Biconditional: $\leftrightarrow$; Exclusive Or: $\bar{\vee}[13])$, which are used in the sequel.

Table 1: Structure Matrices of Some Logical Operators

\begin{tabular}{|c|c||c|c|}
\hline Operator & Structure Matrix & Operator & Structure Matrix \\
\hline$\neg$ & $M_{n}=\delta_{2}\left[\begin{array}{lll}2 & 1\end{array}\right]$ & $\vee$ & $M_{d}=\delta_{2}\left[\begin{array}{llll}1 & 1 & 1 & 2\end{array}\right]$ \\
\hline$\rightarrow$ & $M_{i}=\delta_{2}\left[\begin{array}{llll}1 & 2 & 1 & 1\end{array}\right]$ & $\leftrightarrow$ & $M_{e}=\delta_{2}\left[\begin{array}{llll}1 & 2 & 2 & 1\end{array}\right]$ \\
\hline$\wedge$ & $M_{c}=\delta_{2}\left[\begin{array}{llll}1 & 2 & 2 & 2\end{array}\right]$ & $\bar{\vee}$ & $M_{p}=\delta_{2}\left[\begin{array}{llll}2 & 1 & 1 & 2\end{array}\right]$ \\
\hline
\end{tabular}

\subsection{Boolean (Control) Network}

A Boolean network, $N$, of $n$ elements may be described by its network graph, $G(N)$. $G(N)$ consists of $n$ nodes, denoted by $\mathcal{N}=\left\{x_{1}, \cdots, x_{n}\right\}$, and a set of directed edges $\mathcal{E} \subset \mathcal{N} \times \mathcal{N} . \overline{x_{j}, x_{i}} \in \mathcal{E}$ (i.e., there is an edge from $x_{j}$ to $x_{i}$ ) means $x_{i}$ is affected by $x_{j}$. We refer to Fig. 1 for a network graph (within the rectangular box).

A network graph is not enough to describe a Boolean network completely. A logical dynamical equation is necessary to determine a Boolean network. The dynamics of a Boolean network is expressed 
as

$$
\left\{\begin{array}{l}
x_{1}(t+1)=f_{1}\left(x_{1}, \cdots, x_{n}\right) \\
x_{2}(t+1)=f_{2}\left(x_{1}, \cdots, x_{n}\right) \\
\vdots \\
x_{n}(t+1)=f_{n}\left(x_{1}, \cdots, x_{n}\right), \quad x_{i} \in \mathcal{D},
\end{array}\right.
$$

where $f_{i}, i=1, \cdots, n$ are logical functions. Let $X=\left(x_{1}, \cdots, x_{n}\right)^{T}$ and $F=\left(f_{1}, \cdots, f_{n}\right)^{T}$. Then (2) can briefly denoted as

$$
X(t+1)=F(X(t))
$$

Using the vector form and setting $x(t):=\ltimes_{i=1}^{n} x_{i}(t) \in \Delta^{n}$, and denoting by $M_{i}$ the structure matrix of $f_{i}$, we can express each equation in (2) in a vector form as

$$
x_{i}(t+1)=M_{i} x(t), \quad x_{i} \in \Delta, \quad i=1, \cdots, n .
$$

Finally, we can obtain an algebraic form of (2) (equivalently, (3)) as [3]

$$
x(t+1)=L x(t), \quad x \in \Delta^{n},
$$

where $L \in \mathcal{L}_{2^{n} \times 2^{n}}$ is the transition matrix.

The dynamics of a Boolean control network is expressed as

$$
\left\{\begin{array}{l}
x_{1}(t+1)=f_{1}\left(x_{1}, \cdots, x_{n}, u_{1}, \cdots, u_{m}\right) \\
x_{2}(t+1)=f_{2}\left(x_{1}, \cdots, x_{n}, u_{1}, \cdots, u_{m}\right) \\
\vdots \\
x_{n}(t+1)=f_{n}\left(x_{1}, \cdots, x_{n}, u_{1}, \cdots, u_{m}\right),
\end{array}\right.
$$

where $f_{i}, i=1, \cdots, n$ are logical functions. Similarly, we denote it briefly as

$$
X(t+1)=F(X(t), U(t)), \quad X(t) \in \mathcal{D}^{n}, \quad U(t) \in \mathcal{D}^{m} .
$$

Let $x(t):=\ltimes_{i=1}^{n} x_{i}(t)$ and $u(t):=\ltimes_{i=1}^{m} u_{i}(t)$. We can equivalently express (6) (equivalently, (7)) in an algebraic form as $[4,5]$

$$
x(t+1)=L u(t) x(t), \quad x \in \Delta^{n}, u \in \Delta^{m},
$$

where $L \in \mathcal{L}_{2^{n} \times 2^{n+m}}$ is also called the transition matrix.

We give an example to illustrate them.

Example 2.3 1. In Fig. 1 within the rectangular box we have a Boolean network of four nodes. Assume its dynamics is described by the following equation:

$$
\left\{\begin{array}{l}
A(t+1)=B(t) \vee C(t) \\
B(t+1)=A(t) \leftrightarrow C(t) \\
C(t+1)=A(t) \wedge D(t) \\
D(t+1)=(A(t) \rightarrow B(t)) \bar{\vee} C(t) .
\end{array}\right.
$$


Define $x(t)=A(t) B(t) C(t) D(t)$, it is easy to check that its algebraic form is $x(t+1)=L x(t)$ with

$$
L=\delta_{16}[24571314168833881111] .
$$

(Please refer to the Acknowledgment for a Toolbox, which provides a simple function to calculate this.)

2. Consider the overall Boolean control network in Fig. 1. Say, its dynamics is

$$
\left\{\begin{array}{l}
A(t+1)=(B(t) \vee C(t)) \wedge u_{1} \\
B(t+1)=A(t) \leftrightarrow C(t) \\
C(t+1)=(A(t) \wedge D(t)) \vee u_{2} \\
D(t+1)=(A(t) \rightarrow B(t)) \bar{\vee} C(t) .
\end{array}\right.
$$

Then its algebraic form is $x(t+1)=L u(t) x(t)$, where $L$ can be calculated as

$\begin{array}{rlllllllllllllll}L=\delta_{16}[2 & 2 & 5 & 5 & 1 & 1 & 14 & 14 & 6 & 6 & 1 & 1 & 6 & 6 & 9 & 9 \\ 2 & 4 & 5 & 7 & 1 & 3 & 14 & 16 & 8 & 8 & 3 & 3 & 8 & 8 & 11 & 11 \\ 10 & 10 & 13 & 13 & 9 & 9 & 14 & 14 & 14 & 14 & 9 & 9 & 14 & 14 & 9 & 9 \\ 10 & 12 & 13 & 15 & 9 & 11 & 14 & 16 & 16 & 16 & 11 & 11 & 16 & 16 & 11 & 11] .\end{array}$

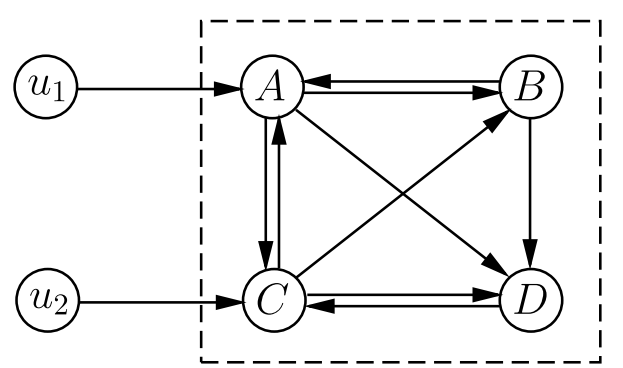

Figure 1: A Boolean (Control) Network

\subsection{Logical Coordinate Transformation}

Definition 2.4 Let $x_{1}, x_{2}, \cdots, x_{n} \in \mathcal{D}$ be a set of independent logical variables. A mapping $\varphi: \mathcal{D}^{n} \rightarrow \mathcal{D}^{n}$, denoted by $Y=\varphi(X)$ and defined by

$$
\varphi:\left\{\begin{array}{l}
y_{1}=g_{1}\left(x_{1}, \cdots, x_{n}\right) \\
\vdots \\
y_{n}=g_{n}\left(x_{1}, \cdots, x_{n}\right)
\end{array}\right.
$$

is called a coordinate transformation, if (11) is a one-to-one and onto mapping.

In vector form we have $x_{i}, y_{i} \in \Delta, \forall i$. Setting $x=\ltimes_{i=1}^{n} x_{i} \in \Delta^{n}$ and $y=\ltimes_{i=1}^{n} y_{i} \in \Delta^{n}$, then the algebraic form of (11) is expressed as

$$
y=T x,
$$


where $T \in \mathcal{L}_{2^{n} \times 2^{n}}$.

Proposition 2.5 [6] (11) is a coordinate transformation, iff the transformation matrix $T$ in (12) is nonsingular.

Note that since $T \in \mathcal{L}_{2^{n} \times 2^{n}}$, when $T$ is nonsingular it becomes an orthogonal matrix [6].

\section{Proposition $2.6[5]$}

1. Consider system (2). Let $z=T x$ be a coordinate transformation. Then the algebraic form (5) of (2) becomes

$$
z(t+1)=T L T^{T} z(t) .
$$

2. Consider system (5). Let $z=T x$ be a coordinate transformation. The algebraic form (8) of (5) becomes

$$
z(t+1)=T L\left(I_{2^{m}} \otimes T^{T}\right) u(t) z(t) .
$$

\subsection{Incidence Matrix}

Assume a Boolean network, $N$ is given, with its nodes $\mathcal{N}=\left\{x_{1}, \cdots, x_{n}\right\}$ and edge set $\mathcal{E}$.

Definition 2.7 An $n \times n$ matrix $\mathcal{I}(N)=\left(b_{i j}\right)$ is called the incidence matrix of $N$, if

$$
b_{i j}= \begin{cases}1, & \overline{x_{j}, x_{i}} \in \mathcal{E}, \\ 0, & \text { otherwise } .\end{cases}
$$

Example 2.8 Consider the network (9), refer to the graph within the rectangular box of Fig. 1. Its incidence matrix is

$$
\left.\mathcal{I}\right|_{(9)}=\left[\begin{array}{llll}
0 & 1 & 1 & 0 \\
1 & 0 & 1 & 0 \\
1 & 0 & 0 & 1 \\
1 & 1 & 1 & 0
\end{array}\right] .
$$

Remark 2.9 1. If a Boolean network $N$ has $n$ nodes, its incidence matrix $\mathcal{I}(N) \in \mathcal{B}_{n \times n}$.

2. The incidence matrix of a Boolean network can also be constructed from its dynamical equations. But you have to be very careful that in dynamic equations there may have some fabricated variables, which should be removed. [5] provided a mechanical procedure to remove all fabricated variables from a logic expression.

\section{Logical Operations on Boolean Matrices}

The main purpose is to define a distance on $\mathcal{B}_{m \times n}$. We first define the logical operators on $\mathcal{B}_{m \times n}$. 
Definition 3.1 1. Let $X=\left(x_{i j}\right) \in \mathcal{B}_{m \times n}$ and $\sigma$ an unary logical operator. Then $\sigma: \mathcal{B}_{m \times n} \rightarrow \mathcal{B}_{m \times n}$ is defined by $\sigma X=\left(\sigma x_{i j}\right)$. For instance,

$$
\neg X:=\left(\neg x_{i j}\right) .
$$

2. Let $X=\left(x_{i j}\right), Y=\left(y_{i j}\right) \in \mathcal{B}_{m \times n}$ and $\sigma$ a binary logical operator. Then $\sigma: \mathcal{B}_{m \times n} \times \mathcal{B}_{m \times n} \rightarrow \mathcal{B}_{m \times n}$ is defined by $X \sigma Y:=\left(x_{i j} \sigma y_{i j}\right)$. For instance,

$$
X \vee Y:=\left(x_{i j} \vee y_{i j}\right), \quad \text { etc. }
$$

3. Let $\alpha \in \mathcal{D}$ and $X=\left(x_{i j}\right) \in \mathcal{B}_{m \times n}$. $\sigma$ is a binary logical operator. Then $\sigma: \mathcal{D} \times \mathcal{B}_{m \times n} \rightarrow \mathcal{B}_{m \times n}$ is defined by $\alpha \sigma X:=\left(\alpha \sigma x_{i j}\right)$. Similarly, $\sigma: \mathcal{B}_{m \times n} \times \mathcal{D} \rightarrow \mathcal{B}_{m \times n}$ is defined by $X \sigma \alpha:=\left(x_{i j} \sigma \alpha\right)$. For instance

$$
\alpha \wedge X=\left(\alpha \wedge x_{i j}\right) ; \quad X \wedge \alpha=\left(x_{i j} \wedge \alpha\right)
$$

Next, we consider the scalar product and the (semi-tensor) product on the set of Boolean matrices.

Definition 3.2 1. Let $\alpha \in \mathcal{D}$. The scalar product of $\alpha$ with $X \in \mathcal{B}_{m \times n}$ is

$$
\alpha \cdot X:=\alpha \wedge X ; \quad X \cdot \alpha:=X \wedge \alpha
$$

Note that since it coincides with the conventional real number product, we use the same product symbol. For compactness, we may also even omit the symbol in the sequel.

2. Let $X=\left(x_{i j}\right) \in \mathcal{B}_{m \times n}$ and $Y \in \mathcal{B}_{p \times q}$ be two Boolean matrices. Then the Kronecker product of $X, Y$ is defined as

$$
X \otimes Y=\left(x_{i j} \cdot Y \mid i=1, \cdots, m ; j=1, \cdots, n\right) \in \mathcal{B}_{m p \times n q} .
$$

3. Let $\alpha, \beta, \alpha_{i} \in \mathcal{D}, i=1,2, \cdots, n$. The Boolean plus is defined as follows.

$$
\left\{\begin{array}{l}
\alpha+{ }_{B} \beta:=\alpha \vee \beta \\
B \sum_{i=1}^{n} \alpha_{i}:=\alpha_{1} \vee \alpha_{2} \vee \cdots \vee \alpha_{n} .
\end{array}\right.
$$

4. Let $X=\left(x_{i j}\right) \in \mathcal{B}_{m \times n}$ and $Y=\left(y_{i j}\right) \in \mathcal{B}_{n \times p}$. Then the Boolean product of Boolean matrices

$$
X \ltimes_{B} Y:=Z \in \mathcal{B}_{m \times p}
$$

where

$$
z_{i j}={ }^{B} \sum_{k=1}^{n} x_{i k} \cdot y_{k j}, \quad i=1, \cdots, m ; j=1, \cdots, p .
$$

5. Let $A \prec_{t} B\left(A \succ_{t} B\right)$. Then the Boolean product of $A, B$ is defined as

$$
A \ltimes_{B} B:=\left(A \otimes I_{t}\right) \ltimes_{B} B . \quad\left(A \ltimes_{B} B:=A \ltimes_{B}\left(B \otimes I_{t}\right) .\right)
$$


6. Assume $A \ltimes_{B} A$ is well defined. Then the Boolean power

$$
A^{(k)}:=\underbrace{A \ltimes_{B} A \ltimes_{B} \cdots \ltimes_{B} A}_{k} .
$$

Note that $\ltimes_{B}$ may be omitted when there is no possible confusion.

We give some simple examples.

Example 3.3 Let

$$
A=\left[\begin{array}{ll}
1 & 1 \\
0 & 1 \\
0 & 0
\end{array}\right] ; \quad B=\left[\begin{array}{cc}
0 & 1 \\
1 & 0 \\
0 & 1
\end{array}\right] ; \quad C=\left[\begin{array}{cc}
0 & 1 \\
1 & 0 \\
1 & 0 \\
0 & 1
\end{array}\right]
$$

Then

$$
\begin{gathered}
\neg A=\left[\begin{array}{ll}
0 & 0 \\
1 & 0 \\
1 & 1
\end{array}\right] ; \quad A \wedge B=\left[\begin{array}{ll}
0 & 1 \\
0 & 0 \\
0 & 0
\end{array}\right] ; \\
A+{ }_{B} B=A \vee B=\left[\begin{array}{ll}
1 & 1 \\
1 & 1 \\
0 & 1
\end{array}\right] ; \quad A \rightarrow B=\left[\begin{array}{ll}
0 & 1 \\
1 & 0 \\
1 & 1
\end{array}\right] ; \\
A \leftrightarrow B=\left[\begin{array}{ll}
0 & 1 \\
0 & 0 \\
1 & 0
\end{array}\right] ; \quad A \bar{\vee} B=\left[\begin{array}{ll}
1 & 0 \\
1 & 1 \\
0 & 1
\end{array}\right] ; \\
A \ltimes_{B} C=\left[\begin{array}{ll}
1 & 1 \\
1 & 1 \\
1 & 0 \\
0 & 1 \\
0 & 0 \\
0 & 0
\end{array}\right] ; \quad B \ltimes_{B} C=\left[\begin{array}{ll}
1 & 0 \\
0 & 1 \\
1 & 0 \\
0 & 1 \\
1 & 0 \\
0 & 1
\end{array}\right] .
\end{gathered}
$$

Next, we define a partial order on $\mathcal{B}_{m \times n}$, and a "distance", called the vector distance on $\mathcal{B}_{m \times n}$.

Definition 3.4 Let $X=\left(x_{i j}\right), Y=\left(y_{i j}\right) \in \mathcal{B}_{m \times n}$. We said $X \leq Y$ if $x_{i j} \leq y_{i j}, \forall i, j$.

Definition 3.5 Let $X=\left(x_{i j}\right), Y=\left(y_{i j}\right) \in \mathcal{B}_{m \times n}$. The vector distance of $X$ and $Y$, denoted by $d(X, Y)$, is defined as

$$
d(A, B)=A \bar{\vee} B .
$$

Since both Boolean product and Boolean plus are order-preserving, it is easy to verify the following properties, which are the generalization of the corresponding results (for vector cases) in [14].

Proposition 3.6 Assume $A \geq B$ and $C \geq D$, then (as long as the product is well defined)

$$
A \ltimes_{B} C \geq B \ltimes_{B} D .
$$


Proposition 3.7 Let $X, Y, Z \in \mathcal{B}_{m \times n}$. The vector distance satisfies

$$
\left\{\begin{array}{l}
d(X, Y)=0 \Leftrightarrow X=Y, \\
d(X, Y)=d(Y, X), \\
d(X, Z) \leq d(X, Y)+{ }_{B} d(Y, Z) .
\end{array}\right.
$$

\section{Global Stability}

This section considers global stability of a Boolean network. That is, when will there exists a fixed point as the unique attractor. Equivalently, when a Boolean dynamics will globally converges to a state. A key tool in this investigation is the vector distance.

Denote $\mathcal{X}=\mathcal{D}^{n}$ as the state space of the Boolean network (2) (or Boolean control network (6)). (In vector form we have $\mathcal{X}=\Delta^{n}$.) $X \in \mathcal{X}$ is expressed as $X=\left(x_{1}, \cdots, x_{n}\right)$. we consider a logical mapping $F: \mathcal{X} \rightarrow \mathcal{X}$, which is described as

$$
\left\{\begin{array}{l}
z_{1}=f_{1}\left(x_{1}, \cdots, x_{n}\right), \\
\vdots \\
z_{n}=f_{n}\left(x_{1}, \cdots, x_{n}\right) .
\end{array}\right.
$$

It is briefly denoted as

$$
Z=F(X), \quad \text { where } X, Z \in \mathcal{X} .
$$

This mapping may come from the Boolean network (2).

Theorem 4.1 [14] Let $X, Y \in \mathcal{X}$. Then

$$
d(F(X), F(Y)) \leq \mathcal{I}(F) \ltimes_{B} d(X, Y),
$$

where $\mathcal{I}(F)$ is the incidence matrix of $F$.

Theorem 4.2 [14] For mapping (27) if there exists a matrix $M \in \mathcal{B}_{n \times n}$ such that

$$
d(F(X), F(Y)) \leq M \ltimes_{B} d(X, Y), \quad \forall X, Y \in \mathcal{X},
$$

then

$$
\mathcal{I}(F) \leq M
$$

Theorem 4.3 [14] Let $E, F: \mathcal{X} \rightarrow \mathcal{X}$ be two logical mappings. Then

$$
\mathcal{I}(E \circ F) \leq \mathcal{I}(E) \ltimes_{B} \mathcal{I}(F) .
$$

An immediate application of the above theorem is

Corollary 4.4 Let $\xi$ be a fixed point of (2). Then

$$
d(X(k), \xi) \leq[\mathcal{I}(F)]^{(k)} \ltimes_{B} d(X(0), \xi) .
$$

Particularly, if the $j_{1}, \cdots, j_{s}$ columns of $[B(F)]^{(k)}$ are zero, and $x_{\alpha}(0)=\xi_{\alpha}, \forall \alpha \notin\left\{j_{1}, \cdots, j_{s}\right\}$, then $x(t)=\xi, t \geq k$. 
It is obvious that if 0 is a fixed point of $F$ and there exists an integer $k>0$ such that $[B(F)]^{(k)}=0$. Then the system globally converges to 0 .

Definition 4.5 System (2) is said to be globally stable if it globally converges to a fixed point. In other words, it has a fixed point as the only attractor.

Example 4.6 Consider the follow system

$$
\left\{\begin{array}{l}
x_{1}(t+1)=f_{1}\left(x_{2}(t), x_{3}(t)\right) \\
x_{2}(t+1)=f_{2}\left(x_{4}(t)\right. \\
x_{3}(t+1)=c_{0} \\
x_{4}(t+1)=f_{4}\left(x_{3}\right)
\end{array}\right.
$$

where $f_{1}, f_{2}$, and $f_{3}$ can be any logical functions, and $c_{0}$ is a logical constant. Briefly, we denote (32) as

$$
X(t+1)=F(X(t)), \quad X \in \mathcal{D}^{4} .
$$

The incidence matrix of $F$ is

$$
\mathcal{I}(F)=\left[\begin{array}{llll}
0 & 1 & 1 & 0 \\
0 & 0 & 0 & 1 \\
0 & 0 & 0 & 0 \\
0 & 0 & 1 & 0
\end{array}\right]
$$

It is easy to check that $[\mathcal{I}(F)]^{(4)}=0$, assume 0 is a fixed point of the system (32), then it globally converges to 0 .

Summarizing the above arguments, we have

Proposition 4.7 Consider system (2) (equivalently, (3)). Assume $0 \in \mathcal{D}^{n}$ is a fixed point of $F$ and there exists an integer $k>0$ such that

$$
[\mathcal{I}(F)]^{(k)}=0
$$

then 0 is the unique global attractor.

Note that if $x_{e}=\left(e_{1}, e_{2}, \cdots, e_{n}\right)$ is a fixed point of the system (2), the above method is still useful for testing whether $x_{e}$ is a global attractor. Make a coordinate transformation

$$
z_{i}= \begin{cases}x_{i}, & e_{i}=0, \\ \neg x_{i}, & e_{i}=1\end{cases}
$$

Now it is easy to convert the system (2) into a system of $z$ as

$$
z(t+1)=\tilde{F}(z(t))
$$

If there exists a $k>0$ such that $[\mathcal{I}(\tilde{F})]^{(k)}=0$, then $x_{e}$ is a global attractor of the system $(2)$.

It is easy to prove that [14] for a Boolean matrix $H \in \mathcal{B}_{n \times n}$ the followings are equivalent: 
- there exists a $k>0$ such that $H^{(k)}=0$;

- there exists a permutation matrix $P$ such that $P^{T} \ltimes_{B} H \ltimes_{B} P$ is a strictly lower triangular (or upper triangular) matrix.

In fact, when $H=\mathcal{I}(F)$ is an incidence matrix, $P$ means a re-ordering of variables.

Unfortunately, this method is sufficient but not necessary. Consider the following example

Example 4.8 Consider the following system

$$
\left\{\begin{array}{l}
x_{1}(t+1)=x_{1}(t) \wedge x_{2}(t) \\
x_{2}(t+1)=x_{1}(t) \wedge\left(\neg x_{2}(t)\right) .
\end{array}\right.
$$

It is easy to check that 0 is its unique attractor. But its incidence matrix is

$$
\mathcal{I}(F)=\left[\begin{array}{ll}
1 & 1 \\
1 & 1
\end{array}\right]
$$

and

$$
[\mathcal{I}(F)]^{(k)}=\mathcal{I}(F) \neq 0, \quad k \geq 1 .
$$

Now what is the necessary and sufficient condition for a Boolean network to be globally convergent. In fact, we have the following conclusion.

Theorem 4.9 The Boolean network (2) is globally convergent, iff there exists a $k>0$ such that

$$
\mathcal{I}\left(F^{k}\right)=0 .
$$

Proof. (Necessity) If the system is globally convergent, then after $T_{r}$ steps (where $T_{r}$ is the transient period [3]) all the states converge to the global attractor $\xi$. So when $k \geq T_{r}(37)$ is true.

(Sufficiency) Now assume (37) is true. Then for any $x$ we have $F^{k}(x)$ that is constant, say,

$$
F^{k}(x)=\xi
$$

Then for any number $t \geq k$,

$$
F^{t}(x)=F^{k}\left(F^{t-k}(x)\right)=\xi
$$

So, the system converges to $\xi$ globally.

Remark 4.10 1. The Proposition 4.7 and the method right following it are practically useful because the size of the incidence matrix is $n \times n$, which is of the order of $O(n)$.

2. In Theorem $4.9 F^{k}$ is not directly computable. It can only be calculated by the algebraic form of $F$, say $L_{F}$, which is of size $2^{n} \times 2^{n}$. So it is difficult to use it if $n$ is not small.

3. From Theorem 4.3 it is clear that

$$
\mathcal{I}\left(F^{k}\right) \leq[\mathcal{I}(F)]^{(k)}, \quad k \geq 1 .
$$

But in general they are not equal. 
Recall Proposition 4.7. In fact, the condition " 0 is a fixed point" is not necessary for stability. Because from (38) one sees that condition (33) assures that $F^{s}$ is constant for $s \geq k$. Say, $F^{s}(x)=\xi, \forall x$ and $s \geq k$. Then the system globally converges to $\xi$. We write it as a corollary.

Corollary 4.11 Consider the system (2). It is globally stable if the condition (33) holds.

Proposition 4.7 is one of the main tools for the stability analysis and stabilizer design. So some further discussions are necessary.

First, we would like to point out that for a Boolean network its incidence matrix $\mathcal{I}(F)$ is coordinatedepending. The following example shows this.

Example 4.12 Consider the following system

$$
\left\{\begin{array}{l}
x_{1}(t+1)=\left[x_{1}(t) \wedge\left(x_{2}(t) \bar{\vee} x_{3}(x)\right)\right] \vee\left(\neg x_{1}(t) \wedge x_{3}(t)\right), \\
x_{2}(t+1)=\left[x_{1}(t) \wedge\left(\neg x_{2}(t)\right)\right] \vee\left(\neg x_{1} \wedge x_{2}\right), \\
x_{3}(t+1)=\left[x_{1}(t) \wedge\left(\neg\left(x_{2}(t) \wedge x_{3}(t)\right)\right)\right] \vee\left[\neg x_{1}(t) \vee\left(x_{2}(t) \vee x_{3}(t)\right)\right] .
\end{array}\right.
$$

Briefly denote it as

$$
x(t+1)=F(x(t)) .
$$

It is easy to check that 0 is a fixed point of (39). For this system its incidence matrix is

$$
\mathcal{I}(F)=\left[\begin{array}{lll}
1 & 1 & 1 \\
1 & 1 & 0 \\
1 & 1 & 1
\end{array}\right]
$$

There is no way to convert it into a strictly lower triangular form with reordering the variables. In algebraic form it is easy to calculate that system (39) can be expressed as

$$
x(t+1)=L x(t)
$$

where $x(t)=x_{1}(t) x_{2}(t) x_{3}(t)$,

$$
L=\delta_{8}\left[\begin{array}{lllllll}
8 & 3 & 1 & 5 & 1 & 5 & 3
\end{array}\right]
$$

Now we consider a coordinate transformation as

$$
\left\{\begin{aligned}
z_{1} & =\left[x_{1} \wedge \neg\left(x_{3}\right)\right] \vee\left[\left(\neg x_{1}\right) \wedge\left(x_{2} \bar{\vee} x_{3}\right)\right] \\
z_{2} & =\left[x_{1} \wedge\left(x_{2} \bar{\vee} x_{3}\right)\right] \vee\left[\left(\neg x_{1}\right) \wedge x_{3}\right] \\
z_{3} & =x_{2}
\end{aligned}\right.
$$

In the vector form, we can easily calculated that

$$
z=z_{1} z_{2} z_{3}=T x
$$

where

$$
T=\delta_{8}\left[\begin{array}{lllllll}
7 & 1 & 6 & 4 & 5 & 3 & 2
\end{array}\right]
$$

Then in $z$ coordinate frame we have

$$
z(t+1)=T L T^{T} z(t):=\tilde{L} z(t),
$$


where $\tilde{L}$ is

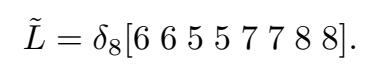

A sequence of $2 \times 2^{n}$ matrices, called the retrievers, have been defined in [5] as

$$
S_{k}^{n}=\delta_{2}[\underbrace{1 \cdots 1}_{2^{n-k}} \underbrace{2 \cdots 2}_{2^{n-k}} \cdots \underbrace{1 \cdots 1}_{2^{n-k}} \underbrace{2 \cdots 2}_{2^{n-k}}], \quad k=1, \cdots, n .
$$

Using them, a mechanical procedure has been proposed in [5] to recover a system from the transition matrix of its algebraic form (42). Next, we recover the system from $\tilde{L}$.

Using retriever $S_{i}^{3}, i=1,2,3$, we have

$$
\left\{\begin{array}{l}
z_{1}(t+1)=M_{1} z(t) \\
z_{2}(t+1)=M_{2} z(t) \\
z_{3}(t+1)=M_{3} z(t)
\end{array}\right.
$$

where

$$
\begin{aligned}
& M_{1}=S_{1}^{3} \tilde{L}=\delta_{2}\left[\begin{array}{llllllll}
2 & 2 & 2 & 2 & 2 & 2 & 2 & 2
\end{array}\right] ; \\
& M_{2}=S_{2}^{3} \tilde{L}=\delta_{2}\left[\begin{array}{llllllll}
1 & 1 & 1 & 1 & 2 & 2 & 2 & 2
\end{array} ;\right. \\
& M_{3}=S_{3}^{3} \tilde{L}=\delta_{2}\left[\begin{array}{lllllll}
2 & 2 & 1 & 1 & 1 & 1 & 2
\end{array}\right]
\end{aligned}
$$

It is easy to convert the matrix form (44) back to logic form, denoted by $z(t+1)=\tilde{F}(z(t))$, as

$$
\left\{\begin{array}{l}
z_{1}(t+1)=0 \\
z_{2}(t+1)=z_{1}(t) \\
z_{3}(t+1)=z_{1}(t) \bar{\vee} z_{2}(t) .
\end{array}\right.
$$

Now consider system (45) (i.e., system (39) under the coordinates Z), its incidence matrix is

$$
\mathcal{I}(\tilde{F})=\left[\begin{array}{lll}
0 & 0 & 0 \\
1 & 0 & 0 \\
1 & 1 & 0
\end{array}\right],
$$

which is strictly lower triangular. We conclude that system (39) globally converges to zero.

Example 4.12 shows that in some cases a coordinate change can help to find a nice incidence matrix to assure the global convergence.

Now a natural question is: If a network is globally stable, can we always find a coordinate transformation such that under new coordinate frame the system has a strict triangular form? Unfortunately, the answer is "No." Let us go back to the Example 4.8. Since $n=2$ there are $\left(2^{2}\right) !=24$ coordinate transformations. We list them in the increasing order as

$$
T_{1}=I_{2} ; \quad T_{2}=\delta_{4}\left[\begin{array}{llll}
1 & 2 & 4 & 3
\end{array}\right] ; \quad T_{3}=\delta_{4}\left[\begin{array}{llll}
1 & 3 & 2 & 4
\end{array}\right] ; \cdots T_{24}=\delta_{4}\left[\begin{array}{llll}
4 & 3 & 2 & 1
\end{array}\right] .
$$


It follows that

$$
\begin{gathered}
T_{2}:\left\{\begin{array} { l } 
{ z _ { 1 } = x _ { 1 } } \\
{ z _ { 2 } = x _ { 1 } \leftrightarrow x _ { 2 } }
\end{array} \quad \Rightarrow \left\{\begin{array}{l}
z_{1}(t+1)=z_{1}(t) \wedge z_{2}(t) \\
z_{2}(t+1)=\neg z_{1}(t) .
\end{array}\right.\right. \\
T_{3}:\left\{\begin{array} { l } 
{ z _ { 1 } = x _ { 2 } } \\
{ z _ { 2 } = x _ { 1 } \leftrightarrow x _ { 2 } }
\end{array} \quad \Rightarrow \left\{\begin{array}{l}
z_{1}(t+1)=z_{1}(t) \wedge z_{2}(t) \\
z_{2}(t+1)=\left(\neg z_{1}(t)\right) \wedge z_{2}(t) .
\end{array}\right.\right. \\
T_{24}:\left\{\begin{array} { l } 
{ z _ { 1 } = \neg x _ { 1 } } \\
{ z _ { 2 } = x _ { 2 } }
\end{array} \Rightarrow \left\{\begin{array}{l}
z_{1}(t+1)=z_{1}(t) \wedge z_{2}(t) \\
z_{2}(t+1)=z_{2}(t) \rightarrow z_{1}(t) .
\end{array}\right.\right.
\end{gathered}
$$

We have plenty of various forms. But unfortunately, no one has incidence matrix in strictly triangular form.

Hence, when the condition of Proposition 4.7 fails even under possible coordinate transformations, we have to invoke Theorem 4.9 .

\section{Stabilization of Boolean Control Networks}

Consider the Boolean control network (6).

Definition 5.1 The global stabilization problem of system (6) is to find, if possible, $\{u(t)\}$ such that the system becomes globally convergent. If $u(t)=W x(t), t=1,2, \cdots$, which is a set of logical functions, then the control is called the state feedback control.

Consider the following example.

Example 5.2 Consider the following system

$$
\left\{\begin{array}{l}
x_{1}(t+1)=x_{3}(t) \vee u \\
x_{2}(t+1)=\neg x_{1}(t) \\
x_{3}(t+1)=x_{1}(t) \leftrightarrow x_{2}(t),
\end{array}\right.
$$

It is obvious that as long as we can delete $x_{3}(t)$ by using control $u$, the system is globally stable because the incidence matrix becomes strictly low triangular.

This is easy to be done. We may choose an open-loop control as $u(t)=1$, or closed-loop control $u(t)=\neg x_{3}(t)$.

To get a general design method, we first consider the expression of logical state variables. Let $x_{1}, \cdots, x_{n}$ be $n$ logical state variables. There are two ways to express $x_{i}$ :

(i) Scalar Form: $x_{i} \in \mathcal{D}, i=1, \cdots, n$. Putting them together, we denote

$$
X=\left(x_{1}, \cdots, x_{n}\right)^{T} \in \mathcal{D}^{n} .
$$

(ii) Vector Form: $x_{i} \in \Delta, i=1, \cdots, n$. Multiplying them together, we denote

$$
x=\ltimes_{i=1}^{n} x_{i} \in \Delta_{2^{n}} .
$$


Define a set of vectors as

$$
s_{k}^{n}=[\underbrace{1 \cdots 1}_{2^{n-k}} \underbrace{0 \cdots 0}_{2^{n-k}} \cdots \underbrace{1 \cdots 1}_{2^{n-k}} \underbrace{0 \cdots 0}_{2^{n-k}}], \quad k=1, \cdots, n .
$$

Remark 5.3 1. $s_{k}^{n} \in \mathcal{B}_{1 \times 2^{n}}, k=1, \cdots, n$. So the logical operators are applicable to them.

2. Comparing (47) with (43) one sees that $s_{k}^{n}$ can be obtained from the first row of $S_{k}^{n}$ by replacing 2 by 0 .

Then we define a matrix as

$$
\mathcal{S}^{n}=\left[\begin{array}{c}
s_{1}^{n} \\
s_{2}^{n} \\
\vdots \\
s_{n}^{n}
\end{array}\right] \in \mathcal{B}_{n \times 2^{n}}
$$

In fact, they are the first row of the corresponding retriever matrices [5].

The following proposition is easily verifiable.

Proposition 5.4 1. From scalar form to vector form, we have

$$
x=\left[\left(x_{1} \leftrightarrow s_{1}^{n}\right) \wedge\left(x_{2} \leftrightarrow s_{2}^{n}\right) \wedge \cdots \wedge\left(x_{n} \leftrightarrow s_{n}^{n}\right)\right]^{T}, \quad \forall x_{i} \in \mathcal{D} .
$$

2. From vector form to scalar form, we have

$$
X=\mathcal{S}^{n} x
$$

Example 5.5 Let $x_{s}=(1,0,1,0)$. Then in vector form we have

$$
\begin{aligned}
x= & {\left[1 \leftrightarrow(1111111100000000)^{T}\right] \wedge\left[0 \leftrightarrow(1111000011110000)^{T}\right] } \\
& \wedge\left[1 \leftrightarrow(1100110011001100)^{T}\right] \wedge\left[0 \leftrightarrow(1010101010101010)^{T}\right] \\
= & (1111111100000000)^{T} \wedge(0000111100001111)^{T} \\
& \wedge(1100110011001100)^{T} \wedge(0101010101010101)^{T} \\
= & (0000010000000000)^{T} .
\end{aligned}
$$

Let $X=\delta_{16}^{9}$. Then

$$
x_{s}=\mathcal{S}^{4} x_{v}=(0,1,1,1) .
$$

Next, we give a systematic analysis on the stabilizer design of Boolean control networks.

First, we define a mapping $\pi: \mathcal{B}_{2^{n} \times 2^{n}} \rightarrow \mathcal{B}_{n \times n}$ as

$$
\begin{aligned}
\pi(L)= & {\left[\left[\left(\mathcal{S}^{n} L\right) \bar{\nabla}\left(\mathcal{S}^{n} L M_{n}\right)\right] \ltimes_{B} \mathbf{1}_{2^{n}},\left[\left(\mathcal{S}^{n} L\right) \bar{\vee}\left(\mathcal{S}^{n} L\right)\left(I_{2} \otimes M_{n}\right)\right] \ltimes_{B} \mathbf{1}_{2^{n}},\right.} \\
& \left.\cdots,\left[\left(\mathcal{S}^{n} L\right) \bar{\nabla}\left(\mathcal{S}^{n} L\right)\left(I_{2^{n-1}} \otimes M_{n}\right)\right] \ltimes_{B} \mathbf{1}_{2^{n}} \ltimes_{B} \mathbf{1}_{2^{n}}\right], \quad L \in \mathcal{B}_{2^{n} \times 2^{n}} .
\end{aligned}
$$

Where $M_{n}$ is the structure matrix of negation (refer to Table 1).

Then we have the following result about how to build the incidence matrix from $L$.

Theorem 5.6 Consider the Boolean network (2) (equivalently, (3)) with its algebraic form (5). The incidence matrix of $F$ can be obtained from $L$ by the following formula:

$$
\mathcal{I}(F)=\pi(L)
$$


Proof. From the construction of $\mathcal{S}^{n}$ it is easy to see that $L_{s}:=\mathcal{S}^{n} L$ is the structure matrix of the system with resulting in scalar form. While $L_{s} M_{n}$ is the structure matrix with $x_{1}$ being replaced by $\neg x_{1}$. If at the $i$-th row they are the same, it means $f_{i}$ is independent of $x_{1}$. Then the $i$-th row of $\left[\left(\mathcal{S}^{n} L\right) \bar{\vee}\left(\mathcal{S}^{n} L M_{n}\right)\right]$ will be identically zero. Hence the $i$-th element of $\left[\left(\mathcal{S}^{n} L\right) \bar{V}\left(\mathcal{S}^{n} L M_{n}\right)\right] \ltimes_{B} \mathbf{1}_{2^{n}} \ltimes_{B} \mathbf{1}_{2^{n}}$ is zero. Otherwise, at least one element in this row is 1 , and hence the $i$-th element of $\left[\left(\mathcal{S}^{n} L\right) \bar{\vee}\left(\mathcal{S}^{n} L M_{n}\right)\right] \ltimes_{B} \mathbf{1}_{2^{n}} \ltimes_{B} \mathbf{1}_{2^{n}}$ is one.

Same argument is applicable to other variables. The only difference is, the negation structure matrix needs to be moved from the front of $x_{i}$ to the front of all variables. Then (51) follows.

We give an example to depict it.

Example 5.7 Assume system (2) has its network transition matrix $L$ as

$$
L=\delta_{16}[19913412121621101419913] \text {. }
$$

A simple routine can calculate that

$$
\mathcal{I}(F)=\pi(L)=\left[\begin{array}{llll}
0 & 0 & 1 & 1 \\
0 & 0 & 1 & 1 \\
1 & 1 & 0 & 0 \\
1 & 1 & 0 & 0
\end{array}\right]
$$

In fact, using the mechanical procedure provided in [5], we can uniquely recover the system from $L$ as

$$
\left\{\begin{array}{l}
x_{1}(t+1)=x_{3}(t) \wedge x_{4}(t) \\
x_{2}(t+1)=x_{3}(t) \vee x_{4}(t) \\
x_{3}(t+1)=x_{1}(t) \rightarrow x_{2}(t) \\
x_{4}(t+1)=x_{1}(t) \leftrightarrow x_{2}(t) .
\end{array}\right.
$$

This verifies the obtained $\mathcal{I}(F)$.

Next, we consider the stabilization problem. Consider system (6) with its algebraic form (8).

Using Theorem 5.6 and Corollary 4.11, a sufficient condition for stabilization with open-loop control is

Lemma 5.8 System (6) is stabilizable by an open-loop control $u$, if $\pi(L u)$ has a strictly lower (or upper) triangular form.

Note that since the incidence matrix is coordinate depended, coordinate transformations have to be taken into consideration.

Using formula (14), we have the following

Theorem 5.9 System (6) (or its algebraic form (8)) is stabilizable by an open-loop control $u$, if there is a coordinate transformation $z=T x$ such that $\pi\left(T L\left(I_{2^{m}} \otimes T^{T}\right) u\right)$ has a strictly lower triangular form.

Note that both possible values of $u$ and coordinate transformations are finite (precisely, $2^{m}$ and $\left(2^{n}\right)$ ! respectively), so theoretically both Lemma 5.8 and Theorem 5.9 are verifiable. 
Example 5.10 Consider the following system

$$
\left\{\begin{array}{l}
x_{1}(t+1)=\neg x_{2}(t) \\
x_{2}(t+1)=\neg x_{4}(t) \leftrightarrow\left(\left(x_{4}(t) \wedge\left(x_{2}(t) \bar{\vee} x_{3}(t)\right)\right) \vee u(t)\right) \\
x_{3}(t+1)=\neg\left(\left(x_{4}(t) \wedge\left(x_{2}(t) \bar{\vee} x_{3}(t)\right)\right) \vee u(t)\right) \\
x_{4}(t+1)=\left(x_{4}(t) \vee\left(x_{2}(t) \bar{\vee} x_{3}(t)\right)\right) \wedge u(t)
\end{array}\right.
$$

After an "try and error" for simplifying the system, we use the following coordinate transformation:

$$
\left\{\begin{array}{l}
z_{1}=x_{4} \\
z_{2}=x_{2} \bar{\nabla} x_{3} \\
z_{3}=\neg x_{3} \\
z_{4}=\neg x_{1}
\end{array}\right.
$$

Its inverse can be easily calculated as

$$
\left\{\begin{array}{l}
x_{1}=\neg z_{4} \\
x_{2}=z_{2} \leftrightarrow z_{3} \\
x_{3}=\neg z_{3} \\
x_{4}=z_{1}
\end{array}\right.
$$

Then the system becomes

$$
\left\{\begin{array}{l}
z_{1}(t+1)=\left(z_{1}(t) \vee z_{2}(t)\right) \wedge u(t) \\
z_{2}(t+1)=\neg z_{1}(t) \\
z_{3}(t+1)=\left(z_{1}(t) \wedge z_{2}(t)\right) \vee u(t) \\
z_{4}(t+1)=z_{2}(t) \leftrightarrow z_{3}(t)
\end{array}\right.
$$

Now it is clear that if we choose

$$
u(t)=0,
$$

the system becomes

$$
\left\{\begin{array}{l}
z_{1}(t+1)=0 \\
z_{2}(t+1)=\neg z_{1}(t) \\
z_{3}(t+1)=\left(z_{1}(t) \wedge z_{2}(t)\right) \\
z_{4}(t+1)=z_{2}(t) \leftrightarrow z_{3}(t)
\end{array}\right.
$$

It is obvious that the incidence matrix of system (59) is

$$
\mathcal{I}(F)=\left[\begin{array}{llll}
0 & 0 & 0 & 0 \\
1 & 0 & 0 & 0 \\
1 & 1 & 0 & 0 \\
0 & 1 & 1 & 0
\end{array}\right],
$$

which is of the strictly lower triangular form. We conclude that the constant control $u(t)=0$ stabilizes system (54). 
Next, we consider the closed-loop control. Let $u(t)$ be a set of logical functions of $x(t)$. Then we can always express it as

$$
u(t)=G x(t)
$$

where $G \in \mathcal{L}_{2^{m} \times 2^{n}}$. Plugging it into (8) yields

$$
x(t+1)=L G x^{2}(t)=L G \Phi_{n} x(t),
$$

where $\Phi_{n}$ is defined in [3] as

$$
\Phi_{n}=\prod_{i=1}^{n} I_{2^{n-1}} \otimes\left[\left(I_{2} \otimes W_{\left[2,2^{n-i}\right]}\right) M_{r}\right]
$$

with $M_{r}=\delta_{4}\left[\begin{array}{ll}1 & 4\end{array}\right]$.

Now the following result is obvious.

Theorem 5.11 System (4) is stabilizable by a closed-loop control $u=G x$, if $\pi\left(L G \Phi_{n}\right)$ has a strictly lower (or upper) triangular form. Moreover, if there exists a coordinate transformation $z=T x$, such that $\pi\left(T L G \Phi_{n} T^{T}\right)$ has a strictly lower (or upper) triangular form, then the control also stabilize the system.

Example 5.12 Consider the system

$$
\left\{\begin{array}{l}
x_{1}(t+1)=\neg x_{2}(t) \\
x_{2}(t+1)=\neg x_{4}(t) \leftrightarrow\left(\left(x_{4}(t) \wedge\left(x_{2}(t) \bar{\vee} x_{3}(t)\right)\right) \vee u(t)\right) \\
x_{3}(t+1)=\neg\left(\left(x_{4}(t) \wedge\left(x_{2}(t) \bar{\vee} x_{3}(t)\right)\right) \vee u(t)\right) \\
x_{4}(t+1)=\left(x_{4}(t) \vee\left(x_{2}(t) \bar{\vee} x_{3}(t)\right)\right) \vee u(t)
\end{array}\right.
$$

In fact, it is obtained from (53) by changing the ways of inputs. Using the same coordinate transformation as in Example 5.10, we have

$$
\left\{\begin{array}{l}
z_{1}(t+1)=\left(z_{1}(t) \vee z_{2}(t)\right) \vee u(t) \\
z_{2}(t+1)=\neg z_{1}(t) \\
z_{3}(t+1)=\left(z_{1}(t) \wedge z_{2}(t)\right) \wedge u(t) \\
z_{4}(t+1)=z_{2}(t) \leftrightarrow z_{3}(t)
\end{array}\right.
$$

One can check that constant (open-loop) controls can not stabilize the system. If we use a closed-loop control

$$
u(t)=\neg z_{1}(t) \wedge \neg z_{2}(t),
$$

the system becomes

$$
\left\{\begin{array}{l}
z_{1}(t+1)=1 \\
z_{2}(t+1)=\neg z_{1}(t) \\
z_{3}(t+1)=0 \\
z_{4}(t+1)=z_{2}(t) \leftrightarrow z_{3}(t)
\end{array}\right.
$$


Obviously, it is globally stable. Converting the control back to the original coordinate frame, we conclude that

$$
u(t)=\neg x_{4}(t) \wedge \neg\left(x_{2}(t) \bar{\vee} x_{3}(t)\right)
$$

stabilizes system (63).

The advantage of using metric-based analysis is that the size of the involved matrix is small. The disadvantage is the condition is only a sufficient one. Next, we search for necessary and sufficient condition.

As discussed in Section 2, the global stability of a free boolean network is equivalent to that there exists a $k>0$ such that $F^{k}=$ constant. In vector form it is equivalent to $L^{k}$ has equal columns, which is the global attractor. An $s \times s$ logic matrix, $M$, is said to be a matrix of constant mapping if there exists a $\delta_{s}^{j}$, such that

$$
\operatorname{Col}(M)=\left\{\delta_{s}^{j}\right\}
$$

Now consider the stabilization by a constant control $u$. Then the control dependent transition matrix is $L u$.

Using the properties of semi-tensor product, it is easy to calculate that

$$
(L u)^{k}=L\left[\left(I_{2^{m}} \otimes L\right) \Phi_{m}\right]^{m-1} u \text {. }
$$

Since $k$ should be less than or equal to the transient time, i.e. $k \leq T_{r} \leq 2^{n}$, we can get an easily verifiable necessary and sufficient condition as follows: Note that $L\left[\left(I_{2^{m}} \otimes L\right) \Phi_{m}\right]^{m-1}$ is a $2^{n} \times 2^{n+m}$ matrix. We split it into $2^{m}$ square blocks as:

$$
L\left[\left(I_{2^{m}} \otimes L\right) \Phi_{m}\right]^{m-1}:=\left[\begin{array}{llll}
L_{1}^{k} & L_{2}^{k} & \cdots & L_{2^{m}}^{k}
\end{array}\right]
$$

Using this notation and according to the above argument, we have the following necessary and sufficient condition.

Theorem 5.13 System (4) is stabilizable by a constant control u, iff there exists a matrix of constant mapping

$$
L_{j}^{k}, \quad 1 \leq k \leq 2^{n}, 1 \leq j \leq 2^{m} .
$$

Moreover, corresponding to each matrix of constant mapping $L_{j}^{k}$ the stabilizing control is $u=\delta_{2^{m}}^{j}$.

The following example illustrates this result.

Example 5.14 Consider the following system

$$
\begin{aligned}
& x_{1}(t+1)=\left(x_{1}(t) \vee x_{2}(t)\right) \wedge u \\
& x_{2}(t+1)=\left(x_{2}(t) \wedge u\right) \rightarrow x_{1} .
\end{aligned}
$$

It is easy to calculate that

$$
L=\delta_{4}\left[\begin{array}{lllllll}
1 & 1 & 2 & 3 & 3 & 3 & 3
\end{array}\right] .
$$

Since $\Phi_{1}=M_{r}$, according to Theorem 5.13 we have to calculate

$$
L\left[\left(I_{2} \otimes L\right) M_{r}\right]^{k}, \quad k \geq 1
$$


to see if we can find a constant mapping block. In fact when $k=2$ we have

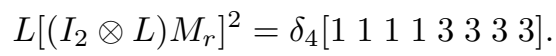

We conclude that if we use control $u=1$ the system is stabilized at $x=\delta_{4}^{1}$ (i.e., $x_{1}=1$ and $x_{2}=1$ ), when $u=0$ the system is stabilized at $x=\delta_{4}^{3}$ (i.e., $x_{1}=0, x_{2}=1$ ).

Consider the state feedback control as in (60). Using the expression (61) and the above argument, the following result is obvious.

Theorem 5.15 System (4) is stabilizable by a closed-loop control $u=G x$, iff there exists a $2^{m} \times 2^{n}$ logical matrix $G$ and an integer $1 \leq k \leq 2^{n}$ such that $\left(L G \Phi_{n}\right)^{k}$ is a matrix of constant mapping.

We give an example.

Example 5.16 Consider the following system

$$
\left\{\begin{aligned}
x_{1}(t+1)= & {\left[x_{2}(t) \vee\left(\neg x_{2}(t) \wedge\left(x_{3}(t) \vee x_{4}(t)\right)\right)\right] \wedge u } \\
x_{2}(t+1)= & \left(x_{2}(t) \wedge\left(x_{3}(t) \vee x_{4}(t)\right)\right) \vee\left[x _ { 1 } ( t ) \wedge \left(\neg x_{2}(t)\right.\right. \\
& \left.\left.\wedge \neg\left(x_{3}(t) \vee x_{4}(t)\right)\right)\right] \\
x_{3}(t+1)= & \left(x_{1}(t) \wedge\left(x_{3}(t) \leftrightarrow x_{4}(t)\right)\right) \vee\left[\neg x _ { 1 } ( t ) \wedge \left(\left(x_{2}(t)\right.\right.\right. \\
& \left.\left.\left.\wedge\left(x_{3}(t) \leftrightarrow x_{4}(t)\right)\right) \wedge\left(\neg x_{2}(t) \wedge\left(x_{3}(t) \wedge x_{4}(t)\right)\right)\right)\right] \\
x_{4}(t+1)= & x_{1}(t) \wedge \neg x_{4}(t) \vee\left[\neg x _ { 1 } ( t ) \wedge \left(\left(x_{2}(t) \wedge \neg x_{4}(t)\right)\right.\right. \\
& \left.\left.\vee\left(\neg x_{2}(t) \wedge \neg\left(x_{3}(t) \rightarrow x_{4}(t)\right)\right)\right)\right]
\end{aligned}\right.
$$

It is easy to verify that if we choose

$$
G=\left[\begin{array}{llll}
1 & 1 & 0 & 0 \\
0 & 0 & 1 & 1
\end{array}\right]^{3}
$$

then

$$
\left(L G \Phi_{4}\right)^{14}=\delta_{16}[\underbrace{16 \cdots 16}_{16}] .
$$

Note that

$$
u(t)=G x(t)=x_{1}(t),
$$

which globally stabilizes system (69) to $x_{1}=0, x_{2}=0, x_{3}=0, x_{4}=0$.

Next, we briefly discuss the case when the system is required to converge to a particular state $x_{0}$. In this case the problem is a little bit simpler. In addition to above stability requirements, we need to assure $x_{0}$ is a fixed point of the control system. We may write it down as a corollary.

Corollary 5.17 1. System (4) is globally stabilized to $x_{0}$ by a constant control $u$, iff $u$ satisfies

$$
\operatorname{Lux}_{0}=x_{0},
$$

and there exists an integer $k>0$, such that $(L u)^{k}$ is a constant mapping. 
2. System (4) is globally stabilized to $x_{0}$ by a constant control $u=G x$, iff $G$ satisfies

$$
L G \Phi_{n} x_{0}=x_{0}
$$

and there exists an integer $k>0$, such that $\left(L G \Phi_{n}\right)^{k}$ is a constant mapping.

Finally, we consider the stabilization by an open-loop control, $u(t)=\ltimes_{i=1}^{m} u_{i}(t), t=1,2, \cdots$. Assume we want to stabilize it to $x_{0}$.

First of all, it is obvious that a necessary condition is, there is a control $u_{e} \in \Delta_{2^{m}}$ such that

$$
L u_{e} x_{0}=x_{0}
$$

Secondly, note that

$$
x\left(t_{0}+k+1\right)=L u\left(t_{0}+k\right) L u\left(t_{0}+k-1\right) \cdots L u\left(t_{0}+1\right) x\left(t_{0}\right) .
$$

To make all trajectories converge to $x_{0}$, there must be a $k>0$ such that

$$
L u(k) L u(k-1) \cdots L u(1) x \equiv x_{0}, \quad \forall x \in \Delta_{2^{n}} .
$$

This is equivalent to

$$
\operatorname{Col}(L u(k) L u(k-1) \cdots L u(1))=\left\{x_{0}\right\}
$$

Observe that

$$
\begin{aligned}
& \operatorname{Lu}(k) L u(k-1) \cdots L u(1) \\
& =L\left(I_{2^{m}} \otimes L\right)\left(I_{2^{2 m}} \otimes L\right) \cdots\left(I_{2^{(k-1) m}} \otimes L\right) \ltimes_{i=k}^{1} u(i) \\
& :=\left[L_{1}^{k}, L_{2}^{k}, \cdots, L_{2^{k m}}^{k}\right] \ltimes_{i=k}^{1} u(i) .
\end{aligned}
$$

Now it is clear that if there is a $1 \leq j \leq 2^{k m}$ such that $L_{j}^{k}$ corresponds to the constant mapping $\psi(x) \equiv x_{0}$, we can choose the control

$$
\ltimes_{i=k}^{1} u(i)=\delta_{2^{m n}}^{j}
$$

such that (73) holds.

Summarizing the above arguments, we have

Theorem 5.18 System (6) is globally stabilized to $x_{0}$ by an open-loop control $u(t), t=1,2, \cdots$, iff

(i) there are an integer $k>0$ and $a L_{j}^{k}, 1 \leq j \leq 2^{k m}$, such that

$$
\operatorname{Col}\left(L_{j}^{k}\right)=\left\{x_{0}\right\}
$$

(ii) there is a $u_{e} \in \Delta_{2^{m}}$ such that (72) holds.

We give an example for this. 
Example 5.19 Consider the following system

$$
\left\{\begin{array}{l}
x_{1}(t+1)=x_{1}(t) \vee u_{1}(t) \\
x_{2}(t+1)=\left(x_{2}(t) \vee x_{3}(t)\right) \leftrightarrow u_{1}(t) \\
x_{3}(t+1)=\left(u_{1}(t) \rightarrow x_{2}(t)\right) \vee x_{3}(t) \\
x_{4}(t+1)=\left(x_{3}(t) \wedge u_{2}(t)\right) \rightarrow x_{4}(t) .
\end{array}\right.
$$

Set $x(t)=\ltimes_{i=1}^{4} x_{i}(t)$ and $u(t)=\ltimes_{i=1}^{2} u_{i}(t)$. Using vector form, (75) can be expressed as

$$
x(t+1)=L u(t) x(t),
$$

where

$$
\begin{aligned}
& L=\delta_{16}\left[\begin{array}{llllllllllllllll}
1 & 2 & 1 & 1 & 1 & 2 & 7 & 7 & 1 & 2 & 1 & 1 & 1 & 2 & 7 & 7
\end{array}\right. \\
& \begin{array}{llllllllllllllll}
1 & 1 & 1 & 1 & 1 & 1 & 7 & 7 & 1 & 1 & 1 & 1 & 1 & 1 & 7 & 7
\end{array} \\
& \begin{array}{llllllllllllllll}
5 & 6 & 5 & 5 & 5 & 6 & 1 & 1 & 13 & 14 & 13 & 13 & 13 & 14 & 9 & 9
\end{array} \\
& \left.\begin{array}{llllllllllllllll}
5 & 5 & 5 & 5 & 5 & 5 & 1 & 1 & 13 & 13 & 13 & 13 & 13 & 13 & 9 & 9
\end{array}\right] .
\end{aligned}
$$

According to (74), we calculate

$$
L\left(I_{2^{2}} \otimes L\right)\left(I_{2^{2 \times 2}} \otimes L\right) \cdots\left(I_{2^{2(k-1)}} \otimes L\right)
$$

to see whether we can find a constant mapping block. In fact when $k=2$ we have

$$
\begin{aligned}
M & =L\left(I_{2^{2}} \otimes L\right) \\
& :=\left[M_{1}, M_{2}, \cdots, M_{16}\right],
\end{aligned}
$$

where

$$
\begin{aligned}
& M=\delta_{16}\left[\begin{array}{llllllllllllllll}
1 & 2 & 1 & 1 & 1 & 2 & 7 & 7 & 1 & 2 & 1 & 1 & 1 & 2 & 7 & 7
\end{array}\right. \\
& \begin{array}{llllllllllllllll}
1 & 1 & 1 & 1 & 1 & 1 & 7 & 7 & 1 & 1 & 1 & 1 & 1 & 1 & 7 & 7
\end{array} \\
& \begin{array}{llllllllllllllll}
1 & 2 & 1 & 1 & 1 & 2 & 1 & 1 & 1 & 2 & 1 & 1 & 1 & 2 & 1 & 1
\end{array} \\
& \begin{array}{llllllllllllllll}
1 & 1 & 1 & 1 & 1 & 1 & 1 & 1 & 1 & 1 & 1 & 1 & 1 & 1 & 1 & 1
\end{array} \\
& \begin{array}{llllllllllllllll}
1 & 1 & 1 & 1 & 1 & 1 & 7 & 7 & 1 & 1 & 1 & 1 & 1 & 1 & 7 & 7
\end{array} \\
& \begin{array}{llllllllllllllll}
1 & 1 & 1 & 1 & 1 & 1 & 7 & 7 & 1 & 1 & 1 & 1 & 1 & 1 & 7 & 7
\end{array} \\
& \begin{array}{llllllllllllllll}
1 & 1 & 1 & 1 & 1 & 1 & 1 & 1 & 1 & 1 & 1 & 1 & 1 & 1 & 1 & 1
\end{array} \\
& \begin{array}{llllllllllllllll}
1 & 1 & 1 & 1 & 1 & 1 & 1 & 1 & 1 & 1 & 1 & 1 & 1 & 1 & 1 & 1
\end{array} \\
& \begin{array}{llllllllllllllll}
5 & 6 & 5 & 5 & 5 & 6 & 1 & 1 & 5 & 6 & 5 & 5 & 5 & 6 & 1 & 1
\end{array} \\
& \begin{array}{llllllllllllllll}
5 & 5 & 5 & 5 & 5 & 5 & 1 & 1 & 5 & 5 & 5 & 5 & 5 & 5 & 1 & 1
\end{array} \\
& \begin{array}{llllllllllllllll}
5 & 6 & 5 & 5 & 5 & 6 & 5 & 5 & 13 & 14 & 13 & 13 & 13 & 14 & 13 & 13
\end{array} \\
& \begin{array}{llllllllllllllll}
5 & 5 & 5 & 5 & 5 & 5 & 5 & 5 & 13 & 13 & 13 & 13 & 13 & 13 & 13 & 13
\end{array} \\
& \begin{array}{llllllllllllllll}
5 & 5 & 5 & 5 & 5 & 5 & 1 & 1 & 5 & 5 & 5 & 5 & 5 & 5 & 1 & 1
\end{array} \\
& \begin{array}{llllllllllllllll}
5 & 5 & 5 & 5 & 5 & 5 & 1 & 1 & 5 & 5 & 5 & 5 & 5 & 5 & 1 & 1
\end{array} \\
& \begin{array}{llllllllllllllll}
5 & 5 & 5 & 5 & 5 & 5 & 5 & 5 & 13 & 13 & 13 & 13 & 13 & 13 & 13 & 13
\end{array} \\
& \begin{array}{llllllllllllllll}
5 & 5 & 5 & 5 & 5 & 5 & 5 & 5 & 13 & 13 & 13 & 13 & 13 & 13 & 13 & 13],
\end{array}
\end{aligned}
$$

and $M_{i} \in \mathcal{L}_{16 \times 16}, i=1, \cdots, 16$. From (77), we know that

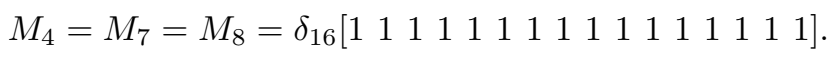


Equivalently,

$$
\operatorname{Col}\left(M_{4}\right)=\operatorname{Col}\left(M_{7}\right)=\operatorname{Col}\left(M_{8}\right)=\left\{\delta_{16}^{1}\right\} .
$$

In the other hand, choosing $u_{e}=\delta_{4}^{1}$ (or $u_{e}=\delta_{4}^{2}$ ), we have

$$
L u_{e} \delta_{16}^{1}=\delta_{16}^{1} .
$$

From Theorem 5.18, system (75) is globally stabilized to $x_{0}=\delta_{16}^{1} \sim(1111)$ by an open-loop control $u(t)$ (or $\bar{u}(t)$ and $\tilde{u}(t)$ ), where

$$
u(t)=\left\{\begin{array}{ll}
\delta_{4}^{4} \sim(00), & t=1 \\
\delta_{4}^{1} \sim(11), & t=2 \\
u_{e}, & t \geq 3,
\end{array} \quad \text { and } \quad \bar{u}(t)=\left\{\begin{array}{ll}
\delta_{4}^{3} \sim(01), & t=1 \\
\delta_{4}^{2} \sim(10), & t=2 \\
u_{e}, & t \geq 3,
\end{array} \quad \text { and } \quad \tilde{u}(t)= \begin{cases}\delta_{4}^{4} \sim(00), & t=1 \\
\delta_{4}^{2} \sim(10), & t=2 \\
u_{e}, & t \geq 3 .\end{cases}\right.\right.
$$

Before ending this section we should like to discuss a little bit more about the conditions in Theorem 5.18. The condition (i) says that all the trajectories can reach the preassigned fixed point $x_{0}$. One may doubt whether the condition (ii) is necessary. In fact, condition (ii) means $x_{0}$ is an equilibrium state under certain control. The following example shows that condition (ii) is also necessary.

Example 5.20 Consider the following system

$$
\left\{\begin{array}{l}
x_{1}(t+1)=\neg\left(x_{1}(t) \wedge u(t)\right) \\
x_{2}(t+1)=\left(u(t) \wedge\left(x_{2}(t) \rightarrow x_{1}(t)\right)\right) \vee\left(\neg u(t) \wedge\left(x_{1}(t) \leftrightarrow x_{2}(t)\right)\right) .
\end{array}\right.
$$

Set $x(t)=\ltimes_{i=1}^{2} x_{i}(t)$. Using the vector form, (75) can be expressed as

$$
\begin{aligned}
x(t+1) & =L u(t) x(t) \\
& =\delta_{4}\left[\begin{array}{llllllll}
3 & 3 & 2 & 1 & 1 & 2 & 2 & 1
\end{array}\right] u(t) x(t) .
\end{aligned}
$$

For any initial state $\xi \in \Delta_{4}$, if we choose $u(1)=\delta_{2}^{2}$, then

$$
\begin{aligned}
x(2) & =L u\left(\begin{array}{ll}
1 \\
)
\end{array}\right. \\
& =\delta_{4}\left[\begin{array}{lll}
1 & 2 & 2
\end{array}\right] \xi .
\end{aligned}
$$

Next, choose $u(2)=\delta_{2}^{1}$, then

$$
\begin{aligned}
& x(3)=\operatorname{Lu}(2) x(2) \\
& =\left(\delta_{4}\left[\begin{array}{llll}
3 & 3 & 2 & 1
\end{array}\right]\right)\left(\delta_{4}\left[\begin{array}{llll}
1 & 2 & 2 & 1
\end{array}\right]\right) \xi \\
& =\delta_{4}\left[\begin{array}{llll}
3 & 3 & 3 & 3
\end{array}\right] \xi \\
& =\delta_{4}^{3}, \quad \forall \xi \in \Delta_{4} \text {. }
\end{aligned}
$$

From (76), it is obvious that there does not exist $u_{e}$, such that

$$
L u_{e} \delta_{4}^{3}=\delta_{4}^{3} .
$$

In step 3, no matter what is the value of $u(3),\left(u(3)=\delta_{2}^{1}\right.$ or $\left.u(3)=\delta_{2}^{2}\right)$, the dynamic of the Boolean network will leave the state $x_{0}=\delta_{4}^{3}$. So, system (75) can not be globally stabilized to $x_{0}$ by an open-loop control $u(t), t=1,2, \cdots$. 


\section{Conclusion}

The stability of Boolean networks and the stabilization of Boolean control networks were investigated in this paper. The semi-tensor product of matrices and the matrix expression of logic provided a powerful tool for this work. The main results consist of two parts. (i) The known results of convergence provided in [14] have been improved by using logical coordinate transformations. Then it has been used to the design of stabilizers for Boolean control networks. (ii) Based on the algebraic form of Boolean (control) networks, necessary and sufficient conditions for stability of Boolean networks were obtained. Then the stabilization of Boolean control networks by constant controls, open-loop controls, and closed-loop controls were investigated and the respective necessary and sufficient conditions were obtained. The stabilizer design techniques were also provided.

Though the results in part (i) are only sufficient, their main advantage is the related computations are less complicated because the incidence matrices used there are of much smaller dimensions $(n \times n)$. The weakness of part (ii) is the computation complexity because they use transition matrices, which are of much larger dimensions $\left(2^{n} \times 2^{n}\right)$.

Acknowledgment An STP toolbox is provided in http://1sc.amss.ac.cn/ dcheng for the related computations.

\section{References}

[1] Akutsu, T., M. Hayashida, W. Ching, M.K. Ng, Control of Boolean networks: hardness results and algorithms for tree structured networks, J. Theoretical Biology, Vol. 244, 670-679, 2007.

[2] Cheng, D., Semi-tensor product of matrices and its applications - A survey, ICCM 2007, Vol. 3, 641-668, 2007.

[3] Cheng, D., H. Qi, A linear representation of dynamics of Boolean networks, IEEE Trans. Aut. Contr., (provisionally accepted).

[4] Cheng, D., Input-state approach to Boolean networks, IEEE Trans. Neural Networks, Vol. 20, No. $3,512-521,2009$.

[5] Cheng, D., H. Qi, Controllability and observability of Boolean networks, Automatica, Vol. 45, No. 7, 1659-1667, 2009.

[6] Cheng, D., Z. Li, H. Qi, Realization of Boolean control networks, Automatica, (to appear).

[7] Davison, E.H., et al., A genomic regulatory network for development. Science 295, 1669-1678, 2002.

[8] Huang, S., I. Ingber, Shape-dependent control of cell growth, differentiation, and apotosis: Switching between attractors in cell regulatory networks, Exp. Cell Res., Vol. 261, 91-103, 2000.

[9] Kauffman, S.A., Metabolic stability and epigenesis in randomly constructed genetic nets, J. Theoretical Biology, Vol. 22, 437-467, 1969.

[10] Kauffman, S.A., The Origins of Order: Self-organization and Selection in Evolution, Oxford University Press, New York, 1993. 
[11] Kauffman, S.A., At Home in the Universe, Oxford University Press, New York, 1995.

[12] S. Onami, K.M. Kyoda, M. Morohashi, H. Kitano, The DBRF method for inferring a gene network from large-scale steady-state gene expression date, in Foundations of Systems Biology, edited by H. Kitano, MIT Press, Cambrige, 59-75, 2001.

[13] Rade, L., B. Westergren, Mathematics Handbook for Science and Engineering, 4th Ed., Studentlitteratur, Sweden, 1998

[14] Robert, F., Discrete Iterations, A Metric Study, Translated by J. Rokne, Springer-Verlag, Berlin, 1986. 